\title{
Individual and flock immunity responses of naïve ducks on smallholder farms after vaccination with H5N1 Avian Influenza vaccine: a study in a province of the Mekong Delta, Vietnam
}

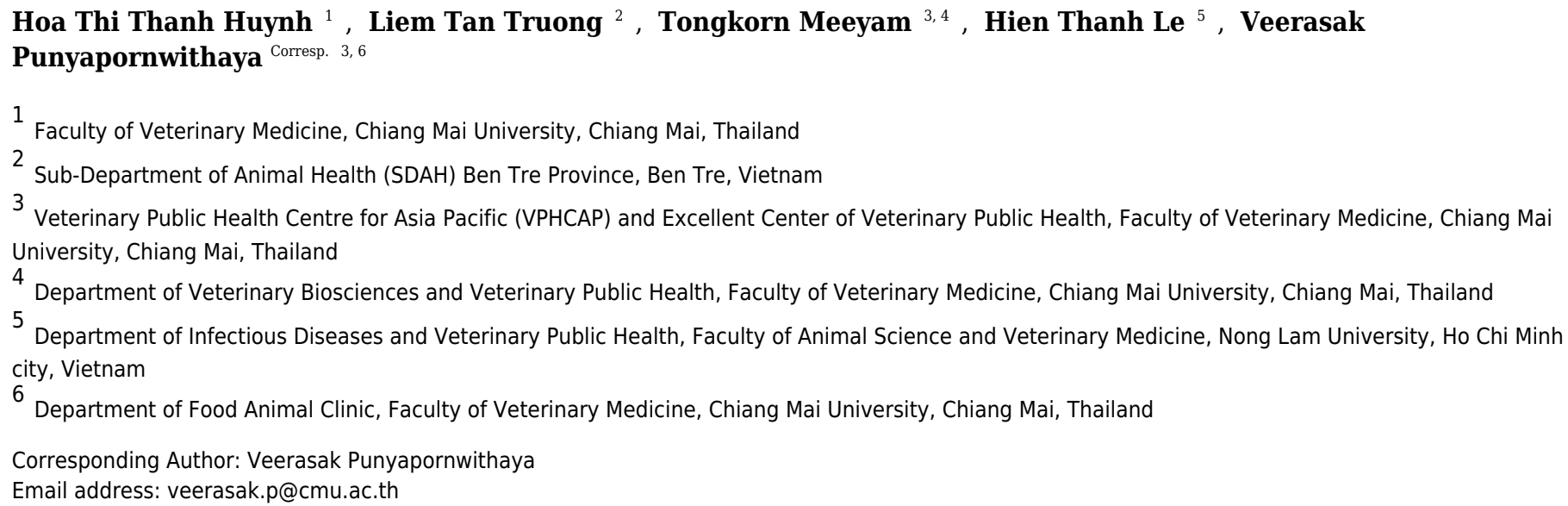

In Vietnam, vaccination has played a crucial role in the national strategy for the prevention and control of $\mathrm{H} 5$ highly pathogenic avian influenza (HPAl). This study aimed to evaluate antibody responses of immunologically naïve domestic ducks to H5N1 avian influenza vaccine currently used in the national mass vaccination program of Vietnam. Blood samples of 166 ducks reared on smallholder farms were individually collected at three sampling time points, namely, right before vaccination, 21 days after primary vaccination, and 21 days after booster vaccination. Vaccine-induced antibody titers of duck sera were measured by the hemagglutination inhibition assay. Temporal differences in mean antibody titers were analyzed using the generalized least-squares method. No sampled ducks showed anti-H5 seropositivity pre-vaccination. The geometric mean titer (GMT) of the vaccinated ducks was 5.30 after primary vaccination, with $80 \%$ of the vaccinated ducks showing seropositivity. This result indicates that the immunity of duck flocks met the targets of the national poultry H5N1 HPAI mass vaccination program. GMT and seropositive rates of the ducks were 6.48 and $96.3 \%$, respectively, after booster vaccination, which were significantly higher than those after primary vaccination. Flocklevel seroprotection rate significantly increased from $68 \%$ to $84.7 \%$, whereas variability in GMT titers decreased from $34.87 \%$ to $26.3 \%$. This study provided important information on humoral immune responses of ducks to the currently used H5N1 vaccine under field conditions. Our findings may help guide veterinary authorities in planning effective vaccine protocols for the prevention and control of H5N1 in the target poultry population. 


\section{Individual and Flock Immunity Responses of Naïve}

2 Ducks on Smallholder Farms after Vaccination with

3 H5n1 Avian Influenza Vaccine: A Study in a Province

4 of the Mekong Delta, Vietnam

5

6

7

8

9

10

11

12

13

14

15

16

17

18

19

20

21

22

23

24

25

26

27

28

29

30

31

32

33

34

35

36

37

38

Hoa Thi Thanh Huynh ${ }^{1}$, Liem Tan Truong ${ }^{2}$, Tongkorn Meeyam ${ }^{3,4}$, Hien Thanh Le ${ }^{5}$, Veerasak Punyapornwithaya ${ }^{3,6}$

${ }^{1}$ Faculty of Veterinary Medicine, Chiang Mai University, Chiang Mai 50100, Thailand

${ }^{2}$ Sub-Department of Animal Health (SDAH), Ben Tre Province, Ben Tre 930000, Vietnam

${ }^{3}$ Veterinary Public Health Centre for Asia Pacific (VPHCAP) and Excellent Center of

Veterinary Public Health, Faculty of Veterinary Medicine, Chiang Mai University, Chiang Mai

50100, Thailand

${ }^{4}$ Department of Veterinary Biosciences and Veterinary Public Health, Faculty of Veterinary Medicine, Chiang Mai University, Chiang Mai 50100, Thailand

${ }^{5}$ Department of Infectious Diseases and Veterinary Public Health, Faculty of Animal Science and Veterinary Medicine, Nong Lam University, Ho Chi Minh City 720400, Vietnam

${ }^{6}$ Department of Food Animal Clinic, Faculty of Veterinary Medicine, Chiang Mai University, Chiang Mai 50100, Thailand

Corresponding Author:

Veerasak Punyapornwithaya ${ }^{3,6}$

Veterinary Public Health Centre for Asia Pacific (VPHCAP) and Excellent Center of Veterinary

Public Health, Faculty of Veterinary Medicine, Chiang Mai University, Chiang Mai 50100,

Thailand

Department of Food Animal Clinic, Faculty of Veterinary Medicine, Chiang Mai University,

Chiang Mai 50100, Thailand

Email address: veerasak.p@.cmu.ac.th

\section{Abstract}

In Vietnam, vaccination has played a crucial role in the national strategy for the prevention and control of H5 highly pathogenic avian influenza (HPAI). This study aimed to evaluate antibody responses of immunologically naïve domestic ducks to $\mathrm{H} 5 \mathrm{~N} 1$ avian influenza vaccine currently used in the national mass vaccination program of Vietnam. Blood samples of 166 ducks reared on smallholder farms were individually collected at three sampling time points, namely, right before vaccination, 21 days after primary vaccination, and 21 days after booster vaccination. Vaccine-induced antibody titers of duck sera were measured by the hemagglutination inhibition assay. Temporal differences in mean antibody titers were analyzed using the generalized least- 
squares method. No sampled ducks showed anti-H5 seropositivity pre-vaccination. The geometric mean titer (GMT) of the vaccinated ducks was 5.30 after primary vaccination, with $80 \%$ of the vaccinated ducks showing seropositivity. This result indicates that the immunity of duck flocks met the targets of the national poultry H5N1 HPAI mass vaccination program. GMT and seropositive rates of the ducks were 6.48 and $96.3 \%$, respectively, after booster vaccination, which were significantly higher than those after primary vaccination. Flock-level seroprotection rate significantly increased from $68 \%$ to $84.7 \%$, whereas variability in GMT titers decreased from $34.87 \%$ to $26.3 \%$. This study provided important information on humoral immune responses of ducks to the currently used $\mathrm{H} 5 \mathrm{~N} 1$ vaccine under field conditions. Our findings may help guide veterinary authorities in planning effective vaccine protocols for the prevention and control of $\mathrm{H} 5 \mathrm{~N} 1$ in the target poultry population.

\section{Introduction}

In Vietnam - a country with a high total poultry population- $-\mathrm{H} 5 \mathrm{~N} 1$ highly pathogenic avian influenza (HPAI) has become endemic, which has resulted in tremendous economic losses to the poultry industry. This disease also poses a considerable threat to public health because it has caused sporadic human infections since its first reported outbreak in 2003 [1-3]. The Vietnamese Government initiated a national poultry mass vaccination program against H5-type HPAI viruses in 2005 after the failure of other anti-HPAI measures such as massive stamping out, movement control, and disinfection [3]. Vaccination has reduced the number of HPAI infections and outbreaks among poultry and has consequently reduced the risk of human exposure and number of human cases; these results are important steps toward the prevention and control of HPAI [4]. The Re-6 vaccine (A/duck/Guangdong/1332/2010 H5N1 clade 2.3.2) has been extensively used to immunize poultry since 2014 . This vaccine contains antigens with close antigenic similarity to the $\mathrm{H} 5 \mathrm{~N} 1$ virus subclade $2.3 .2 .1 \mathrm{c}$, which is widely circulated in southern Vietnamese provinces [5]. The vaccine has been used in Vietnam until now since it has been showing a certain protective effect against H5N1 HPAI to the poultry population.

Domestic ducks represent the second largest poultry population in Vietnam following chickens. Duck populations contribute to the maintenance and dissemination of the H5-type HPAI viruses because they are natural reservoirs of this virus [6,7]. Therefore, in Vietnam, mass immunization of ducks is a part of the disease control strategy [8]. The Mekong Delta (MKD) has a large population and high density of domestic ducks and is at high risk of H5N1 HPAI outbreaks because of the presence of large numbers of backyard or smallholder poultry farms $[9$, 10]. The small-scale poultry production system has several characteristics that have made it a primary site of H5N1 HPAI viral infections. The problem of inappropriate vaccination is prevalent among smallholder poultry farms. Moreover, farm owners lack sufficient knowledge of poultry diseases and, consequently, show poor compliance to vaccination guidelines [11]. Many ducks on smallholder farms in MKD have not been administered with the recommended twodose vaccination regimen; instead, local farmers implement the single-dose vaccination regimen. Similarly, rates of compliance to the recommended two-dose vaccination regimen for meat-type 
ducks are low in southwestern regions [12]. Whether the desired protective antibody response can be induced by the single-dose vaccination regimen warrants further inquiry, and the benefit of the two-dose vaccination regimen should be confirmed. Nevertheless, published data on the effectiveness of vaccines against avian influenza (AI), particularly the Re-6 vaccine, in inducing antibody response in domestic ducks under field conditions remain limited $[10,13]$. This limitation, in turn, restricts the availability of information that may guide veterinary authorities in improving the national H5N1 HPAI vaccination strategy of Vietnam. Kandeil et al., in 2017, assessed the immunogenicity and vaccine efficacy against an Egyptian H5N1 clade 2.2.1.2 virus in different avian hosts in backyard conditions [14].

In this study, we aimed to evaluate the effectiveness of the Re-6 vaccine against H5N1 HPAI in immunologically naïve domestic ducks reared on smallholder farms. We evaluated the effectiveness of the vaccine in inducing immunological responses under field conditions by examining the levels and variation of antibody responses at individual and flock levels.

\section{Materials \& Methods}

\section{Sample size}

Sample size was determined using power analysis and sample size software (PASS version 15, NCSS, LLC. Utah, USA; http://www.ncss.com/software/pass/) on the basis of statistical analysis with repeated measured data. A total of 166 vaccinated ducks from 11 smallholder duck farms were included. Five ducks from each farm received saline instead of the vaccine (control). In total, 20 ducks, which were individually identified using leg bands, were sampled from each farm.

\section{Study design}

The study was conducted in two districts in a province of MKD, southern Vietnam, from July 2017 to December 2017. No H5-type HPAI outbreaks have been reported in the province since 2014. Ducks aged 18-20 days were selected from 11 farms with the support of the SubDepartment of Animal Health (SDAH). The 11 farms represented various flock sizes (approximately 100-1,300 ducks per farm), production purposes (meat or layer ducks), and duck breeds. Each selected duck was vaccinated twice. Blood samples of each duck were collected at three separate time points, namely, right before vaccination, 21 days after primary vaccination (21 dpv), and 21 days after booster vaccination ( $21 \mathrm{dpbv})$. The first blood sample was immediately collected before primary vaccination. Pre-vaccination sampling was performed to detect H5-specific antibodies that were potentially derived from maternal immunity or natural infection.

Vaccination was performed with the inactivated reassortant $\mathrm{H} 5 \mathrm{~N} 1$ avian influenza vaccine, Re-6 strain, which expresses the HA antigen of A/duck/Guangdong/1332/2010 H5N1 clade 2.3.2 (HA titer $\geq 1: 256$ before deactivation). This strain is the only anti-H5N1 HPAI vaccine used for mass immunization in the province in which this study was conducted. Vaccines were intramuscularly administered in the breast using automatic syringes. Each duck 
119 received 0.5 and $1 \mathrm{~mL}$ of the primary and booster vaccines, respectively. Except for five control

120

121

122

123

124

125

126

127

128

129

130

131

132

133

134

135

136

137

138

139

140

141

142

143

144

145

146

147

148

149

150

151

152

153

154

155

156

157

158

ducks, the remainder of the flock was vaccinated.

Briefly, $1-2 \mathrm{~mL}$ of blood was drawn from each duck through the medial metatarsal vein.

Serum was separated from blood by centrifugation. In addition to blood samples, pooled tracheal swab samples were collected from five vaccinated ducks from each farm at the final sampling time point. The swabs were sent to the Regional Animal Health Office VI (RAHO VI) and tested via real-time reverse transcriptase-polymerase chain reaction (RRT-PCR) to detect H5-type HPAI viruses circulating in the sampled farms during the observation period.

\section{Serological assay for the detection of H5-specific antibodies in duck sera}

H5-specific antibodies in vaccinated duck sera were detected and quantified by the hemagglutination inhibition (HI) assay in V-bottom microtiter plates with two-fold dilutions, $0.5 \%$ specific pathogen-free chicken red blood cells (RBCs), and 4 hemagglutination units (HAU) of antigen derived from the H5N1 virus strain AlCklScot159 (RAA 7002-APHA Scientific, Surrey, UK). Tests were conducted by the SDAH of the province (license number LAS-NN 59; ISO/IEC 17025: VILAT-0043) in accordance with their routine HPAI postvaccination serosurveillance method. A reference positive serum with a known titer and a negative control serum were included in each test plate. Before the serological assay, duck sera were heat-inactivated at $56^{\circ} \mathrm{C}$ for $30 \mathrm{~min}$ and then treated with $10 \%$ chicken $\mathrm{RBC}$ suspension for removing nonspecific inhibitors to prevent the occurrence of nonspecific HA reactions in the sera of nonchicken species during the HI assay.

The HI titer of a sample is the reciprocal of the highest serum dilution that causes complete inhibition of HA activity of RBCs. HI titers were reported as $\log _{2}$ titers $[15,16]$ for compatibility with results obtained through the routine post-vaccination sero-monitoring program. The starting dilution for the HI assay was 1:8 $\left(3 \log _{2}\right)$. Samples with HI titer $<4$ were considered seronegative, whereas those with $\mathrm{HI}$ titer $\geq 4$ were considered seropositive. These thresholds are in compliance with Vietnam's national regulation on post-vaccination surveillance for H5N1 HPAI (MARD-DAH, 487/TY-DT, 2009), which is based on the OIE Manual [17]. For calculating antibody geometric mean titers (GMTs), samples without detectable antibody levels (HI titer $<3 \log _{2}$ ) were assigned an HI titer of $2 \log _{2}$. Seropositive rates $(\%)$ were calculated with the cutoff level of $4 \log _{2}$, and seroprotection rates (\%) were calculated starting from $5 \log _{2}$, following the criteria set in the OIE Manual [18].

\section{Ethics statement}

This study was approved by the Institutional Ethical Review Board of Hanoi University of Public Health (IRB-HUPH, approval number 308/2017/YTCC-HD3). The IRB was registered with the U.S. Department of Health and Human Services (IORG number 0003239, FWA number FWA00009326). Permission to conduct the study was obtained from the SDAH of the province where this study was conducted. The SDAH also collaborated on this project. 
159

160

161

162

163

164

165

166

167

168

169

170

171

172

173

174

175

176

177

178

179

180

181

182

183

184

185

186

187

188

189

190

191

192

193

194

195

196

197

198

\section{Statistical analysis}

Antibody titers were transformed into $\log _{2}$ values, as mentioned above, prior to further analysis. Descriptive data of HI antibody titers were presented by individual farms and by the time of sample collection ("time"). GMT [GMT \pm standard error (SE)], coefficient of variation $(\% \mathrm{CV}$, presenting variation in antibody titers, $\% \mathrm{CV}=100 \times$ standard deviation $/ \mathrm{mean}), \%$ seropositive rate, and $\%$ seroprotection rate were calculated.

Temporal differences in GMT were compared using the generalized least-squares (GLS) method for repeated measurements using R statistical software version 3.3.2 [19] with the nlme package [20]. A mixed model with GLS was constructed and fitted using the restricted maximum likelihood estimation method. GMT of antibodies was defined as the dependent variable. "Farm" and "time" were defined as fixed effects, whereas "individual duck" was defined as random effect. Various mixed models were constructed with different covariance structures, including compound symmetry, general correlation matrix, and autoregressive process of order1 (ar1) [20]. Values of the Akaike information criterion (AIC) for each model were then compared to identify the best-fit model. The model with compound symmetry covariance structure had the lowest AIC value. Thus, results of this model were interpreted. Residuals from the fitted model were tested for normality by plotting standardized residuals against quintiles of the standard normal as well as for homogeneity of variance by plotting standardized residuals against fitted values. Tukey's test was used for multiple comparisons when mean differences were significant. Seropositive rates after primary and booster vaccinations were compared using the proportionality test. The level of significance for statistical analysis was set at $\alpha=0.05$.

\section{Results}

\section{Anti-H5 HI antibody titers}

To investigate whether maternal antibodies have already decreased and active infections have not occurred, pre-vaccination $\mathrm{HI}$ titer levels of the ducks were evaluated. No sampled ducks were positive for pre-vaccination anti-H5 antibodies (HI titer $<4)$. The control ducks did not exhibit detectable antibody levels throughout the observation period. Notably, RRT-PCR revealed that the H5N1 virus was undetected in the samples. Moreover, H5-type HPAI outbreaks did not occur in the study areas during the observation period, and the vaccination did not result in adverse effects or illnesses among duck flocks. Thus, the vaccine was well tolerated by the ducks.

HI assay results for antibody responses after each of the two H5N1 HPAI vaccinations are summarized in Table 1 . Nearly $17 \%$ of the vaccinated ducks $(n=28 / 166)$ did not respond to primary vaccination (HI titers $<4$ ), whereas more than $70 \%$ showed antibody responses with HI titers between 4 and 7 . Booster vaccination increased antibody titers, and almost $73 \%$ of the vaccinated ducks $(n=119 / 164)$ showed HI titers between 6 and 9 . Thus, increased HI titers are the dominant humoral immune responses of ducks to each dose of the Re- 6 vaccine.

Antibody titers increased over time. GMT after booster vaccination was significantly higher than that after primary vaccination (Table 1). The highest difference in GMTs was 
199

200

201

202

203

204

205

206

207

208

209

210

211

212

213

214

215

216

217

218

219

220

221

222

223

224

225

226

227

228

229

230

231

232

233

234

235

236

237

238

$2.8 \log _{2}$. This increasing trend was observed on all sampled farms (Fig. 1). In addition, considerable variations were observed in antibody responses at 21 days after primary vaccination. Minor variations in antibody responses following booster vaccination were observed on all sampled farms (Fig. 1, Tables $1 \& 2$ ).

\section{Seropositive rates and seroprotection rates}

Pre-vaccination, no sampled duck tested positive for H5N1 HPAI antibodies. Booster vaccination significantly increased seropositive and seroprotection rates of the vaccinated ducks $(\mathrm{p}<0.01)$ (Table 1). Seropositive rates following booster vaccination exceeded $80 \%$ on all farms. Similarly, seroprotection rates increased after booster vaccination (Table 2).

\section{Discussion}

This study was conducted to evaluate antibody responses of immunologically naïve ducks reared on smallholder farms. The vaccination protocol used in this study is similar to that currently applied by governmental veterinary services in the ongoing mass vaccination program against H5N1 HPAI.

A potential limitation of this study is that because of the evolutionary dynamics of H5type HPAI virus clades, the strain $\mathrm{A} \backslash \mathrm{Ck} \backslash \mathrm{Scot} \backslash 59 \mathrm{H} 5$ antigen might be suboptimally antigenically matched to the Re-6 vaccine strain. However, because of nonscientific considerations of the DAH, the strain AlCk $\backslash$ Scot $159 \mathrm{H} 5$ has been the only one licensed and widely used for anti-H5 antibody detection in routine HPAI post-vaccination serosurveillances and commercial tests in veterinary diagnostic laboratories throughout the country until now. The main objective of postvaccination sero-monitoring programs is to estimate the proportion of poultry with anti-H5 antibodies. Therefore, due to the fact that several other vaccines are used in different regions of the country, the Scot/59 antigen strain has been considered reasonably effective in evaluating antibody titers induced by vaccinations. Therefore, for this study to have a practical significance that its results can be comparable with those of other relevant studies conducted in Vietnam and those obtained through routine post-vaccination sero-monitoring programs, we used the Scot/59 antigen in our study.

Given the variations in characteristics of the household farming sector, ducks may exhibit different responses to the vaccination protocol applied by the governmental veterinary services. Therefore, this study included 11 smallholder farms to represent variations in farm characteristics. Meat and layer ducks were included. Most included ducks were mixed breeds. Considering large variations in flock sizes, management practices, and other factors related to sampled farms, we could not stratify farms based on flock size or management type. Therefore, individual farms were included in the GLS model as a fixed effect.

HA-specific antibody titers measured by the $\mathrm{HI}$ assay were the principal indicators of vaccine-induced protective immunity against H5N1 HPAI viruses [21, 22]. Mean antibody titers within the poultry population are expected to increase following vaccination. In this study, the two-dose vaccination regimen stimulated antibody response in ducks. Pre-vaccination, the ducks 
239 lacked HA-specific antibodies. However, numerous ducks showed immune responses after 240 primary vaccination. GMT values significantly increased after booster vaccination compared 241 with those after primary vaccination. After primary vaccination, the desired overall GMT was 242 achieved despite the lack of seroconversion in some vaccinated ducks. GMT reported in this 243 study was higher than that reported in previous studies conducted in other provinces of MKD, 244 involving poultry vaccinated with the same vaccine. One study has reported a GMT value of 2451.63 in 28-day-old ducks [23], whereas another has reported a GMT value of 3.32 in 35-day-old 246 ducks after primary vaccination [24]. In a study conducted in the Tien Giang province, GMT 247 values of 1.7, 3.4, 4.3, and 4.45 have been reported in 15-, 45-, 75-, and 105-day-old chickens, 248 respectively, after primary vaccination [25]. Differences in mean antibody titers reported in the 249

250 251

252 253 254 255 256 257 258 259 260 present study and those reported in previous studies may be attributed to differences in schedules of vaccination and post-vaccination sample collection. Ducks in this study were vaccinated and sampled at an older age than those in other studies and, thus, produced stronger responses to primary vaccination.

Notably, booster immunization increased GMTs, reflecting the effect of vaccination. In addition, the proportion of ducks with high $\mathrm{HI}$ titers increased on every sampled farm. The results correspond to two studies conducted under field conditions in other countries. For instance, the previously mentioned study in Egypt revealed that the antibody titer levels markedly increased after the booster dose [14]. Also, it was reported that H5-type virus vaccination increased seroconverted proportions after a booster vaccination in 13 member countries of the European Union [11]. These results are also consistent with findings of Bertelsen and Lecu et al. that suggested that the two-dose immunization regimen remarkedly elevates the $\mathrm{HI}$ antibody titer levels in birds [26, 27].

Because this study involved several duck farms, within- and between-farm variations in GMTs were observed (Fig. 1). First, ducks from the same farm showed different GMTs after primary vaccination because some ducks exhibited a seroconversion response, whereas some did not. This result may be attributed to various endogenous factors, such as differences in specific immune reaction, health status, or prevailing disease situation $[28,11]$. Notably, this result may account for the broad range of vaccine-induced HI titers detected in this study, which corresponds with values reported by Phan et al. [23]. However, most ducks that failed to exhibit responses to primary vaccination showed seropositivity after booster vaccination. Veterinary authorities use the extent of variability of antibody response, which is commonly presented by $\% \mathrm{CV}$, as an index to evaluate the effectiveness of a vaccination program. For a majority of poultry diseases, $\% \mathrm{CV}$ should not exceed $40 \%$ after a correct vaccine is administered [29]. High $\% \mathrm{CV}$ values obtained in the present study provided evidence for considerable variation in antibody responses of ducks after primary vaccination. Some ducks showed high HI titers, whereas some showed low HI titers or even seronegativity. These results are consistent with findings of Tarigan et al. who reported that outcomes of field $\mathrm{H} 5 \mathrm{~N} 1$ vaccination were highly variable and farm-related. Specifically, HI titers of individual birds in each flock differed from those of birds in other flocks [30]. Second, GMTs varied on the farm level (Fig. 1, Table 2); this 
279 result may be attributed to differences in field conditions, which may be associated with

280 environmental factors and rearing practices, immunization techniques, vaccine storage, 281 vaccinator's skill and incentive, and other factors that vary across farms [31]. Booster 282 vaccination reduced within- and between-farm variation in antibody responses. Decreased 283 variability in antibody responses following booster immunization has important implications in 284 terms of the effectiveness of the vaccination program.

285 The most important goal of the H5N1 HPAI vaccination program is flock immunity, 286 which is proportional to the level of protection achieved by all birds in a vaccinated flock. 287 Achievement of flock-level immunity is used to evaluate the effectiveness of HPAI vaccination 288 programs. In Vietnam, the national regulation stipulates that flock-level immunity is achieved if $28970 \%$ of the poultry in each flock demonstrates seroconversion (HI titers $\geq 4$ ) and if $80 \%$ of the 290 poultry flocks in each province or region shows flock-level immunity (MARD-DAH, Circular 291 No. 07/2016/TT-BNNPTNT). In this study, primary and booster vaccinations provided some 292 level of protection to most vaccinated ducks when the majority of antibody responses exceeded 293 the cutoff level of $4 \log _{2}(83.13 \%$ and $96.34 \%$ after primary and booster vaccinations, 294 respectively). This finding may partly explain the fact that although local farmers often 295 implement the single-dose vaccination regimen for their flocks, HPAI outbreaks have not 296 occurred in the province since 2014 when the vaccine was first introduced. However, although 297 seropositive rates considerably varied between farms, the overall seropositive rate achieved in 298 this study at 21 days after primary vaccination (83\%) was higher than that previously reported by 299 Pham (2015) (68.18\%) [24] and Phan \& Tran (2016) (33.33\%-40\%) [23] for the same vaccine. 300 Henning (2011) [10] and Tung (2013) [31] have reported low seropositive rates following 301 primary vaccination with different strains.

Booster vaccination provided a higher level of immunity than primary vaccination. In all

304

305

306

307

308

309

310

311

312

313

314

315

316

317

318 sampled farms in this study, booster vaccination produced higher seropositive rates than primary vaccination. The overall seropositive rate of more than $96 \%$ detected following booster immunization corresponds with the observation of provincial veterinary authorities in 2017 and reported rates by Pham (2015) [24] and Phan \& Tran (2016) [23]. In terms of practical significance, results obtained after 21 days of booster vaccination may reflect the vaccineinduced serological immunity of ducks immediately before the common completion time of meat-type duck production cycle in the field, i.e., 63-day-old birds.

Although the minimum protective antibody titer of 1:16 $\left(4 \log _{2}\right)$ has been reported in Vietnam and four other countries worldwide [11], the OIE Manual recommends that the minimum HI serological titer of birds under the field conditions should be 1:32 $\left(5 \log _{2}\right)$ for achieving a good probability of protection against mortality from HPAI infection [18]. Nearly $85 \%$ of the vaccinated ducks in this study showed an antibody levels $\geq 5 \log _{2}$ after booster vaccination, whereas approximately $68 \%$ showed these levels after primary vaccination. These results indicate that ducks undergoing booster vaccination have $17 \%$ increased probability of being protected from mortality in an outbreak. This finding was similar to previous reports that suggested that more than one vaccination dose is required to induce protective immunity and 
319 prevent H5N1 HPAI transmission in ducks and other poultry in field conditions [32, 33, 34]. In 320 addition, Lecu et al. also demonstrated that the administration of booster vaccination to zoo birds

321

322

323

324

325

326

327

328

329

330

331

332

333

334

335

336

337

338

339

340

341

342

343

344

345

346

347

348

349

350

351

352

353

354

355

356

357

in France is necessary to increase mean titers to a protective level [27].

Our findings are expected to reflect the current situation in the study area because we employed materials, serological assay procedures, expression and interpretation methods, and evaluation criteria similar to those used in the national post-vaccination surveillance program. Therefore, our results may guide veterinary authorities in Vietnam in their efforts to improve the effectiveness of the national $\mathrm{H} 5 \mathrm{~N} 1$ vaccination program.

\section{Conclusions}

Primary and booster vaccinations are immunogenic and could induce antibody responses in ducks at levels that meet the targets of the national mass vaccination program. Our results support the notion that compared with the single-dose immunization regimen, the two-dose immunization regimen more intensely induces protective antibody production and, thus, provides better serological immunity against the HPAI virus in ducks. Furthermore, the single-dose vaccination regimen is suitable for short-lived meat ducks, whereas two-dose vaccination regimen is suitable for long-lived ducks, such as layers or breeders, to increase their protective humoral immunity and strengthen flock immunity. Further studies on the duration of antibody responses induced by the single-dose vaccination regimen are warranted. Furthermore, variations in antibody responses of vaccinated ducks suggest that the effectiveness of vaccination varies under different field conditions, which warrant additional attention.

\section{Acknowledgements}

The authors gratefully acknowledge the Veterinary Diagnostic Laboratory and Treatment Division of the SDAH of Ben Tre Province, Vietnam for their collaboration in farm visits, sample collection, and laboratory work.

\section{References}

1. Bui, V. N., Ogawa, H., Trinh, D. Q., Nguyen, T. H., Pham, N. T., Truong, D. A., Bui, A. N., Runstadler, J., Imai, K. and Nguyen, K. V., Genetic characterization of an H5N1 avian influenza virus from a vaccinated duck flock in Vietnam. Virus Genes, 2014. 49(2): p. 278-85. 2. Desvaux, S., Grosbois, V., Pham, T., Fenwick, S., Tollis, S., Pham, N., Tran, A. and Roger, F., Risk factors of highly pathogenic avian influenza H5N1 occurrence at the village and farm levels in the Red River Delta Region in Vietnam. Transboundary and Emerging Diseases, 2011. 58(6): p. 492-502.

3. Tran, C. C., Yanagida, J. F., Saksena, S. and Fox, J., An Alternative Vaccination Approach for The Prevention of Highly Pathogenic Avian Influenza Subtype H5N1 in The Red River Delta, Vietnam -A Geospatial-Based Cost-Effectiveness Analysis. Journal of Veterinary Science, 2016. 3(1): p. 6. 
358 4. Swayne, D.E., Impact of vaccines and vaccination on global control of avian influenza. 359 Avian Diseases, 2012. 56(4 Suppl): p. 818-28.

$3605 . \quad$ Le, T.H. and N.T. Nguyen, Evolutionary dynamics of highly pathogenic avian influenza $361 \mathrm{~A} / \mathrm{H} 5 \mathrm{~N} 1 \mathrm{HA}$ clades and vaccine implementation in Vietnam. Clinical and Experimental Vaccine 362 Research, 2014. 3(2): p. 117-27.

363 6. Hulse-Post, D. J., Sturm-Ramirez, K. M., Humberd, J., Seiler, P., Govorkova, E. A., 364 Krauss, S., Scholtissek, C., Puthavathana, P., Buranathai, C., Nguyen, T. D., Long, H. T., 365 Naipospos, T. S., Chen, H., Ellis, T. M., Guan, Y., Peiris, J. S. and Webster, R. G., Role of 366 domestic ducks in the propagation and biological evolution of highly pathogenic H5N1 influenza 367 viruses in Asia. Proceedings of the National Academy of Sciences of the United States of 368 America, 2005. 102(30): p. 10682-7.

369 7. Swayne, D.E. and D.R. Kapczynski. Avian Influenza, ed. D.E. Swayne. 2008: John 370 Wiley \& Sons. p. 407-51.

$3718 . \quad$ Cha, R. M., Smith, D., Shepherd, E., Davis, C. T., Donis, R., Nguyen, T., Nguyen, H. D., 372 Do, H. T., Inui, K., Suarez, D. L., Swayne, D. E. and Pantin-Jackwood, M., Suboptimal 373 protection against $\mathrm{H} 5 \mathrm{~N} 1$ highly pathogenic avian influenza viruses from Vietnam in ducks 374 vaccinated with commercial poultry vaccines. Vaccine, 2013. 31(43): p. 4953-60.

375 9. Food and Agriculture Organizations of United Nations (FAO), Duck Farming Systems 376 and Avian Influenza in the Mekong Delta of Vietnam, by Bui, X.M., in FAO Smallholder 377 Poultry Production Paper No. 1. 2010.

378 10. Henning, J., Henning, K. A., Morton, J. M., Long, N. T., Ha, N. T., Vu le, T., Vu, P. P., 379 Hoa, D. M. and Meers, J., Highly pathogenic avian influenza (H5N1) in ducks and in-contact 380 chickens in backyard and smallholder commercial duck farms in Viet Nam. Preventive 381 Veterinary Medicine, 2011. 101(3-4): p. 229-40.

382 11. Swayne, D.E., Assessment of national strategies for control of high-pathogenicity avian 383 influenza and low-pathogenicity notifiable avian influenza in poultry, with emphasis on vaccines 384 and vaccination. Revue scientifique et technique (International Office of Epizootics), 2011. 385 30(3): p. 839-70.

386 12. Cuong, N. V., Truc, V. N., Nhung, N. T., Thanh, T. T., Chieu, T. T., Hieu, T. Q., Men, N. 387 T., Mai, H. H., Chi, H. T., Boni, M. F., van Doorn, H. R., Thwaites, G. E., Carrique-Mas, J. J. 388 and Hoa, N. T., Highly Pathogenic Avian Influenza Virus A/H5N1 Infection in Vaccinated Meat 389 Duck Flocks in the Mekong Delta of Vietnam. Transboundary and Emerging Diseases, 2016. 390 63(2): p. 127-35.

391 13. McLaws, M., Priyono, W., Bett, B., Al-Qamar, S., Claassen, I., Widiastuti, T., Poole, J., 392 Schoonman, L., Jost, C. and Mariner, J., Antibody response and risk factors for seropositivity in 393 backyard poultry following mass vaccination against highly pathogenic avian influenza and 394 Newcastle disease in Indonesia. Epidemiology and Infection, 2015. 143(8): p. 1632-42.

395 14. Kandeil, A., Mostafa, A., El-Shesheny, R., El-Taweel, A. N., Gomaa, M., Galal, H., 396 Kayali, G. and Ali, M. A., Avian influenza H5N1 vaccination efficacy in Egyptian backyard 397 poultry. Vaccine, 2017. 35(45): p. 6195-6201. 
398 15. Ferreira, H. L., Pirlot, J. F., Reynard, F., van den Berg, T., Bublot, M. and Lambrecht, B., 399 Immune responses and protection against $\mathrm{H} 5 \mathrm{~N} 1$ highly pathogenic avian influenza virus induced 400 by the Newcastle disease virus H5 vaccine in ducks. Avian Diseases, 2012. 56(4 Suppl): p. 9404018.

402 16. Cagle, C., Wasilenko, J., Adams, S. C., Cardona, C. J., To, T. L., Nguyen, T., Spackman, 403 E., Suarez, D. L., Smith, D., Shepherd, E., Roth, J. and Pantin-Jackwood, M. J., Differences in 404 pathogenicity, response to vaccination, and innate immune responses in different types of ducks 405 infected with a virulent H5N1 highly pathogenic avian influenza virus from Vietnam. Avian 406 Diseases, 2012. 56(3): p. 479-87.

407 17. OIE, Chapter 2.3.4 Avian Influenza, in Terrestrial Manual. 2009.

408 18. OIE, Chapter 2.3.4 Avian Influenza, in Terrestrial Manual. 2017.

409 19. Team, R.D.C., R: A language and environment for statistical computing. R Foundation 410 for Statistical Computing, Vienna, Austria, 2016.

411 20. Pinheiro J, B.D., DebRoy S, and D Sarkar, NLME: Linear and nonlinear mixed effects 412 models. 2015.

413 21. Suarez, D.L. and S. Schultz-Cherry, Immunology of avian influenza virus: a review.

414 Developmental \& Comparative Immunology, 2000. 24(2-3): p. 269-83.

415 22. Sitaras, I., Rousou, X., Kalthoff, D., Beer, M., Peeters, B. and de Jong, M. C. M., Role of 416 vaccination-induced immunity and antigenic distance in the transmission dynamics of highly 417 pathogenic avian influenza H5N1. Journal of The Royal Society Interface, 2016. 13(114): p. 41820150976.

419 23. Phan, C.T. and N.B. Tran, Survey on protective immune responses in ducks vaccinated 420 by Navet-Vifluvac and H5N1 Re-6 vaccine in Hau Giang province. Can Tho University Science 421 Journal, 2016. 44(B): p. 127-31.

422 24. Pham, N.V., Prevalence and humoral immune response of ducks and Muscovy ducks 423 against H5N1 strain Re-6 vaccine in Kien Giang province in Faculty of Agriculture and Applied 424 Biology. 2015, Can Tho University.

425 25. Tran, V.T., An evaluation of antibody response to two avian influenza vaccines in 426 chickens of Tre domestic species in household farms in Cho Gao district, Tien Giang province.

427 Journal of Sciene \& Education of the Southern Agriculture College, 2016. 6: p. 58-64.

428 26. Bertelsen, M. F., Klausen, J., Holm, E., Grondahl, C. and Jorgensen, P. H., Serological 429 response to vaccination against avian influenza in zoo-birds using an inactivated $\mathrm{H} 5 \mathrm{~N} 9$ vaccine. 430 Vaccine, 2007. 25(22): p. 4345-9.

431 27. Lecu, A., De Langhe, C., Petit, T., Bernard, F. and Swam, H., Serologic response and 432 safety to vaccination against avian influenza using inactivated $\mathrm{H} 5 \mathrm{~N} 2$ vaccine in zoo birds. J Zoo 433 Wildl Med, 2009. 40(4): p. 731-43.

434 28. Marangon, S. and L. Busani, The use of vaccination in poultry production. Revue 435 Scientifique et Technique-Office International des Epizooties, 2007. 26(1): p. 265.

436 29. Greenacre, C.B. and T.Y. Morishita, Backyard poultry medicine and surgery: a guide for 437 veterinary practitioners. 2014: John Wiley \& Sons. 
438 30. Tarigan, S., Wibowo, M. H., Indriani, R., Sumarningsih, S., Artanto, S., Idris, S., Durr, P.

439 A., Asmara, W., Ebrahimie, E., Stevenson, M. A. and Ignjatovic, J., Field effectiveness of highly 440 pathogenic avian influenza H5N1 vaccination in commercial layers in Indonesia. PLoS One, 441 2018. 13(1): p. e0190947.

442 31. Tung, D. H., Van Quyen, D.,Nguyen, T.,Xuan, H. T.,Nam, T. N. and Duy, K. D., 443 Molecular characterization of a H5N1 highly pathogenic avian influenza virus clade 2.3.2.1b 444 circulating in Vietnam in 2011. Veterinary Microbiology, 2013. 165(3-4): p. 341-8.

445 32. Swayne, D.E., Avian influenza. 2009: John Wiley \& Sons.

446 33. van der Goot, J. A., van Boven, M., de Jong, M. C. and Koch, G., Effect of vaccination 447 on transmission of HPAI H5N1: the effect of a single vaccination dose on transmission of highly 448 pathogenic avian influenza H5N1 in Peking ducks. Avian Diseases, 2007. 51(1 Suppl): p. 323-4. 449 34. Pantin-Jackwood, M.J. and D.L. Suarez, Vaccination of domestic ducks against H5N1 450 HPAI: a review. Virus Research, 2013. 178(1): p. 21-34. 


\section{Table $\mathbf{1}$ (on next page)}

Humoral immunity of vaccinated ducks at 21 days after primary vaccination ( $21 \mathrm{dpv}$ ) and 21 days after booster vaccination ( $21 \mathrm{dpbv}$ )

$\mathrm{N}=$ total number of ducks in each observation GMT = geometric mean titer $\left(\log _{2}\right)$ of total number of vaccinated ducks \%CV = coefficient of variation, indicating the level of variability of $\mathrm{HI}$ titers. Seropositive $=\mathrm{HI}$ titers $\geq 4$; Seroprotection $=\mathrm{HI}$ titers $\geq 5^{* \text { ab }}$ Values between rows with differing superscripts denote differences in mean or in proportion $(p<0.05)$ 
1 Humoral immunity of vaccinated ducks at 21 days after primary vaccination ( $21 \mathrm{dpv}$ ) and 221 days after booster vaccination ( 21 dpbv)

3 Mean, variability of HI titers, proportion of vaccinated ducks showing seropositivity, and

4 proportion of vaccinated ducks showing seroprotection are presented as values of GMT, \%CV,

5 seropositive rate (\%), and seroprotection rate (\%), respectively.

\begin{tabular}{|c|c|c|c|c|c|c|c|c|c|c|c|c|c|}
\hline \multirow{2}{*}{ Time } & \multirow{2}{*}{$\mathbf{N}$} & \multicolumn{8}{|c|}{ HI titer distribution $\left(\log _{2}\right)$} & \multirow{2}{*}{$\begin{array}{l}\text { GMTs } \\
(\text { mean } \pm \text { SE) }\end{array}$} & \multirow{2}{*}{$\begin{array}{l}\text { CVs } \\
(\%)\end{array}$} & \multirow{2}{*}{$\begin{array}{c}\text { Seropositive } \\
\text { rates }\end{array}$} & \multirow{2}{*}{$\begin{array}{c}\text { Seroprotection } \\
\text { rates }\end{array}$} \\
\hline & & $<3$ & 3 & 4 & 5 & 6 & 7 & 8 & 9 & & & & \\
\hline $21 \mathrm{dpv}$ & 166 & 21 & 7 & 25 & 31 & 33 & 32 & 14 & 3 & 5.30 & 34.87 & 8 & 68 \\
\hline 21 dpbv & 164 & 1 & 5 & 19 & 20 & 38 & 32 & 24 & 25 & $6.48 \pm 0.13^{b}$ & 26.30 & $96.30^{\mathrm{b}}$ & $84.76^{\mathrm{b}}$ \\
\hline
\end{tabular}

6

$7 \mathrm{~N}=$ total number of ducks in each observation

$8 \mathrm{GMT}=$ geometric mean titer $\left(\log _{2}\right)$ of total number of vaccinated ducks

$9 \% \mathrm{CV}=$ coefficient of variation, indicating the level of variability of HI titers.

10 Seropositive $=$ HI titers $\geq 4$; Seroprotection $=$ HI titers $\geq 5$

$11 *$ ab Values between rows with differing superscripts denote differences in mean or in proportion (p

$12<0.05)$ 


\section{Table 2 (on next page)}

Seropositive and seroprotection rates of ducks from the 11 sampled farms at 21 days after primary vaccination ( $21 \mathrm{dpv}$ ) and 21 days after booster vaccination ( $21 \mathrm{dpbv}$ )

*Seropositive rate $=$ the proportion of seropositive vaccinated ducks (HI titers $\geq 4)$

**Seroprotection rate $=$ the proportion of vaccinated ducks with $\mathrm{HI}$ titers at levels that

protect against mortality from HPAI infection in accordance with the OIE's recommendation

(HI titers $\geq 5$ ) 
1 Seropositive and seroprotection rates of ducks from the 11 sampled farms at 21 days after 2 primary vaccination ( $21 \mathrm{dpv}$ ) and 21 days after booster vaccination ( $21 \mathrm{dpbv})$

\begin{tabular}{|c|c|c|c|c|}
\hline $\begin{array}{c}\text { Far } \\
\text { m }\end{array}$ & $\begin{array}{c}\text { Seropositive } \\
\text { rate* } \\
21 \mathrm{dpv}\end{array}$ & $\begin{array}{c}\text { Seropositive } \\
\text { rate } \\
21 \text { dpbv }\end{array}$ & $\begin{array}{c}\text { Seroprotection } \\
\text { rate**} \\
21 \mathrm{dpv}\end{array}$ & $\begin{array}{c}\text { Seroprotection } \\
\text { rate } \\
21 \mathrm{dpbv}\end{array}$ \\
\hline A & 73.33 & 92.86 & 53.33 & 92.86 \\
\hline B & 66.67 & 86.67 & 33.33 & 40.00 \\
\hline $\mathrm{C}$ & 93.33 & 100.00 & 86.67 & 93.33 \\
\hline $\mathrm{D}$ & 73.33 & 100.00 & 60.00 & 100.00 \\
\hline$E$ & 86.67 & 100.00 & 80.00 & 93.33 \\
\hline $\mathrm{F}$ & 93.33 & 100.00 & 86.67 & 100.00 \\
\hline G & 87.50 & 100.00 & 75.00 & 53.33 \\
\hline $\mathrm{H}$ & 86.67 & 100.00 & 80.00 & 100.00 \\
\hline I & 60.00 & 80.00 & 60.00 & 73.33 \\
\hline $\mathrm{J}$ & 93.33 & 100.00 & 93.33 & 93.33 \\
\hline $\mathrm{K}$ & 100.00 & 100.00 & 40.00 & 93.33 \\
\hline
\end{tabular}

3

$4 *$ Seropositive rate $=$ the proportion of seropositive vaccinated ducks (HI titers $\geq 4)$

$5 * *$ Seroprotection rate $=$ the proportion of vaccinated ducks with HI titers at levels that protect

6 against mortality from HPAI infection in accordance with the OIE's recommendation (HI titers $\geq$ 7 5) 
Figure 1

Distribution of $\mathrm{HI}$ titers against the H5 HPAl virus by individual farm after primary and booster vaccinations.

Farms are coded from A to K. Sampling times are referred to as "time," and " $21 \mathrm{dpv}$ " and "21 dpbv" represent $\mathrm{HI}$ results at 21 days after the primary vaccination and 21 days after the booster vaccination, respectively. GMT is represented by $\mathbf{\Delta}$ in the middle of each box.

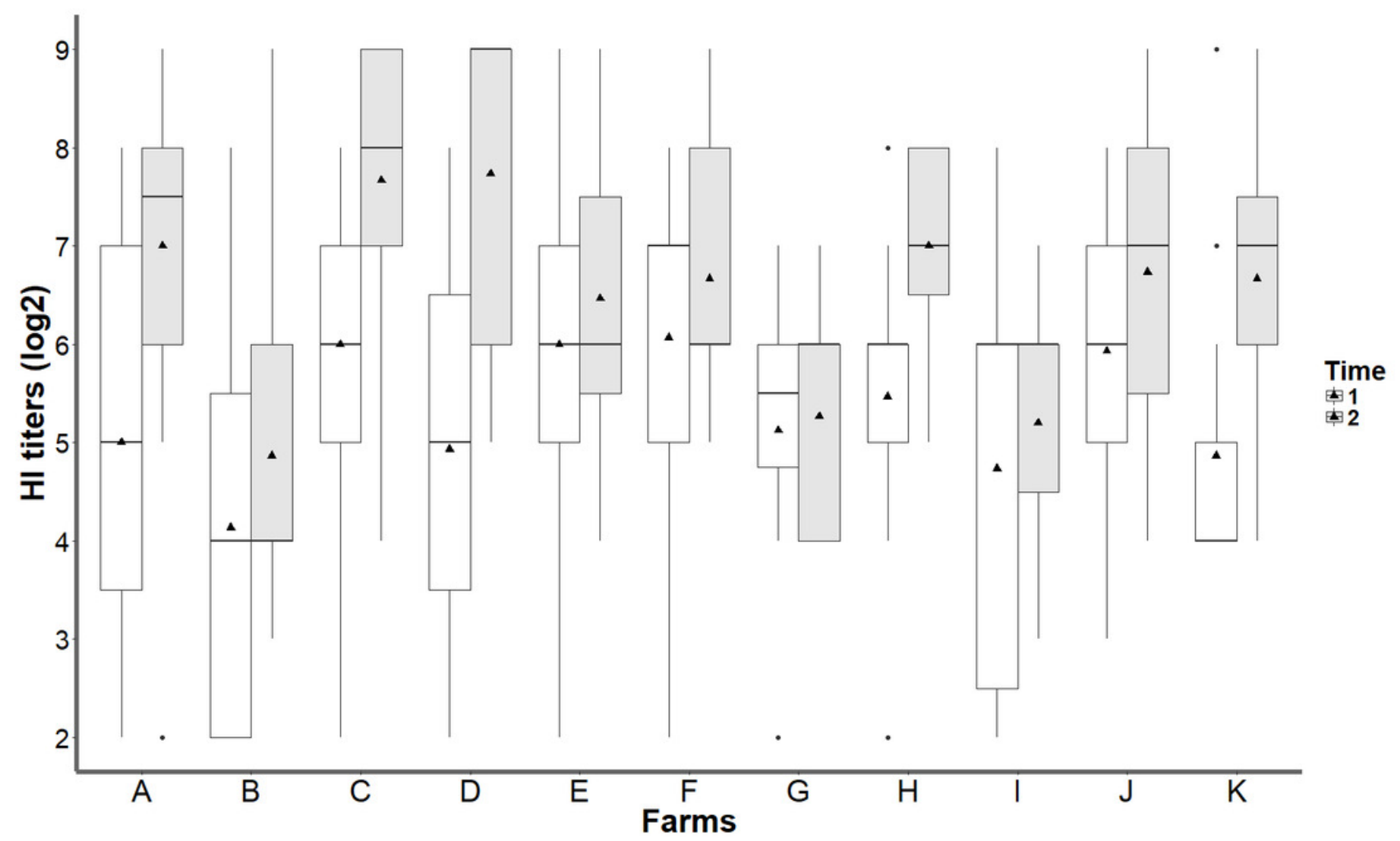

